\title{
The 16 T Dipole Development Program for FCC and HE-LHC
}

\author{
D. Schoerling, D. Arbelaez, B. Auchmann, M. Bajko, A. Ballarino, E. Barzi, G. Bellomo, M. Benedikt, S. Izquierdo \\ Bermudez, B. Bordini, L. Bottura, L. Brouwer, P. Bruzzone, B. Caiffi, S. Caspi, A. Chakraborti, E. Coatanea, G. de \\ Rijk, M. Dhalle, M. Durante, P. Fabbricatore, S. Farinon, H. Felice, A. Fernandez, I.S. Fernandez, P. Gao, B. Gold, \\ T. Gortsas, S. Gourlay, M. Juchno, V. Kashikhin, C. Kokkinos, S. Kokkinos, K. Koskinen, F. Lackner, C. Lorin, K. \\ Loukas, A. Louzguiti, K. Lyytikainen, S. Mariotto, M. Marchevsky, G. Montenero, J. Munilla, I. Novitski, T. Ogitsu, A. \\ Pampaloni, J. C. Perez, C. Pes, C. Petrone, D. Polyzos, S. Prestemon, M. Prioli, A. M. Ricci, J. M. Rifflet, E. Rochepault, \\ S. Russenschuck, T. Salmi, I. A. Santillana, F. Savary, C. Scheuerlein, M. Segreti, C. Senatore,M. Sorbi, M. Statera, A. \\ Stenvall, L. Tavian, T. Tervoort, D. Tommasini, F. Toral, R. Valente, G. Velev, A. P. Verweij, S. Wessel, F. Wolf, \\ F. Zimmermann, and A. V. Zlobin
}

Manuscript received October 26, 2018; accepted February 17, 2019.

This work was supported in part by the European Union's Horizon 2020 research and innovation programme under Grant 654305, EuroCirCol project. Work at LBNL was supported by the Director, Office of Science of the U.S. Department of Energy (DOE) under Contract DE-AC02-05CH11231. Work at NHMFL was supported by the U.S. DOE Office of High Energy Physics (OHEP) under Grant DE-SC0010421. Work at FNAL was supported by Fermi ResearchAl-liance, LLC, under Contract DE-AC02-07CH11359 with the U.S. Department of Energy (OHEP). (Corresponding author: Daniel Schoerling.)

D. Schoerling, M. Bajko, A. Ballarino, M. Benedikt, S. Izquierdo Bermudez, B. Bordini, L. Bottura, G. de Rijk, I. S. Fernandez, F. Lackner, A. M. Louzguiti, J. C. Perez, C. Petrone, M. Prioli, S. Russenschuck, I.A. Santillana, F. Savary, C. Scheuerlein, L. Tavian, D. Tommasini, A. P. Verweij, F. Wolf, and F. Zim-mermann are with the European Organization for Nuclear Research (CERN), Geneva 1211, Switzerland (e-mail: daniel.schoerling@cern.ch; marta.bajko@ cern.ch; amalia.ballarino@cern.ch; michael.benedikt@cern.ch; susana._izquierdo.bermudez@cern.ch; bernardo.bordini@cern.ch; luca.bottura@ cern.ch; gijs.derijk@cern.ch; inigo.sancho.fernandez@cern.ch;_ friedrich._lackner@cern.ch; alexandre.mehdi.louzguiti@cern.ch).

G. Bellomo, S. Mariotto, M. Sorbi, M. Statera, and R. Valente are with the Istituto Nazionale di Fisica Nucleare (INFN), Milano 20133, Italy (e-mail: giovanni.bellomo@mi.infn.it; samuele.mariotto@mi.infn.it; mmartchevskii@ lbl.gov).

M. Dhalle, P. Gao, and S. Wessel are with the University of Twente, Twente 7500, The Netherlands (e-mail: m.m.j.dhalle@utwente.nl; p.gao@utwente.nl).

M. Durante, H. Felice, C. Lorin, C. Pes, J. M. Rifflet, E. Rochepault, and M. Segreti are with the CEA, Saclay 91400, France (e-mail: maria.durante@cea.fr; helene.felice@cea.fr; clement.lorin@cea.fr).

B. Caiffi, P. Fabbricatore, S. Farinon, A. Pampaloni, and A. M. Ricci are with the Istituto Nazionale di Fisica Nucleare (INFN), Genova 16146, Italy (e-mail:barbara.caiffi@ge.infn.it; stefania.farinon@ge.infn.it).

fabbric@ge.infn.it;

A. Fernandez, J. Munilla, and F. Toral are with the Centre for Energy, Envi-ronment and Technology (CIEMAT), Madrid 28040, Spain (e-mail: alejandro.fernandez@ciemat.es; javier.munilla@ciemat.es).

T. Ogitsu is with KEK, Tsukuba 305-0801, Japan (e-mail: toru.ogitsu@ kek.jp).

B. Auchmann and G. Montenero are with the Paul Scherrer Insti-tut (PSI), Villigen 5232, Switzerland (e-mail: bernhard.auchmann@psi.ch; giuseppe.montenero@psi.ch).

A. Chakraborti, E. Coatanea, K. Koskinen, K. Lyytikainen, T. Salmi, and A. Stenvall are with the Tampere University of Applied Sciences (TAMK), Tam-pere 33100, Finland (e-mail: ananda.chakraborti@tut.fi; eric.coatanea@tut.fi; kari.t.koskinen@tut.fi; kari.lyytikainen@tut.fi).

C. Senatore is with the Faculty of Science, University of Geneva (UoG), Geneva 1211, Switzerland.
Abstract-A future circular collider (FCC) with a center-ofmass energy of $100 \mathrm{TeV}$ and a circumference of around $100 \mathrm{~km}$, or an energy upgrade of the $\mathrm{LHC}$ (HE-LHC) to $27 \mathrm{TeV}$ require bending magnets providing $16 \mathrm{~T}$ in a $50-\mathrm{mm}$ aperture. Several development programs for these magnets, based on $\mathrm{Nb}_{3} \mathrm{Sn}$ technology, are being pursued in Europe and in the U.S. In these programs, cos-theta, block-type, common-coil, and canted-costheta magnets are ex-plored; first model magnets are under manufacture; limits on con-ductor stress levels are studied; and a conductor with enhanced characteristics is developed. This paper summarizes and discusses the status, plans, and preliminary results of these programs.

Index Terms-FCC, $\mathrm{Nb}_{3} \mathrm{Sn}$, superconducting, $16 \mathrm{~T}$.

\section{INTRODUCTION}

THE Future Circular Collider (FCC) and the High-Energy Large Hadron Collider (HE-LHC), an LHC energy-doubler, aim at reaching $100 \mathrm{TeV}$ and $27 \mathrm{TeV}$, respectively. The

D. Arbelaez, L. Brouwer, S. Caspi, S. Gourlay, M. Juchno, M. Martchevsky, and S. Prestemon are with Lawrence Berkeley National Laboratory (LBNL), Berkely, CA 94720 USA (e-mail: darbelaez@lbl.gov; lnbrouwer@lbl.gov; s_caspi@lbl.gov; sagourlay@lbl.gov; mjuchno@lbl.gov).

E. Barzi, V. Kashikhin, I. Novitski, G. Velev, and A. V. Zlobin are with Fermi National Accelerator Laboratory (FNAL), Batavia, IL 60510 USA (email: barzi@fnal.gov; vadim@fnal.gov; novitski@fnal.gov).

P. Bruzzone is with Ecole Polytechnique Fed'erale' de Lausanne (EPFL), Lau-sanne 1015, Switzerland (e-mail: pierluigi.bruzzone@psi.ch).

B. Gold and T. Tervoort are with Eidgenossische" Technische Hochschule Zurich," Zurich" 8092, Switzerland (e-mail: barbara.gold@mat.ethz.ch).

T. Gortsas, C. Kokkinos, S. Kokkinos, K. Loukas, and D. Polyzos are with the Department of Mechanical Engineering \& Aeronautics, Univer-sity of Patras, Patras 26504, Greece (e-mail: thodoris.gortsas@gmail.com; charilaos.kokkinos@feacomp.com;_ sotiris.kokkinos@feacomp.com; konstanti-nos.loukas@feacomp.com).

Color versions of one or more of the figures in this paper are available online at http://ieeexplore.ieee.org.

Digital Object Identifier 10.1109/TASC.2019.2900556

This manuscript has been authored by Fermi Research Alliance, LLC under Contract No. DE-AC02-07CH11359 with the U.S. Department of Energy, Office of Science, Office of High Energy Physics. 
TABLE I

Main Target Parameters of the Baseline Cos-Theta Dipole MAgNet For FCC AND HE-LHC

\begin{tabular}{|c|c|c|}
\hline Item & Unit & Value \\
\hline Number of units FCC/HE-LHC & - & $4668 / 1232$ \\
\hline Operating field & $\mathrm{T}$ & 16 \\
\hline Coil physical aperture & $\mathrm{mm}$ & 50 \\
\hline Operating current & $\mathrm{A}$ & 11441 \\
\hline Operating temperature & $\mathrm{K}$ & 1.9 \\
\hline Magnetic length at $1.9 \mathrm{~K}$ FCC $\&$ HE- & $\mathrm{mm}$ & 14069 \\
\hline LHC & & \\
\hline Stored energy at $16 \mathrm{~T}(2$ apertures $)$ & $\mathrm{MJ}$ & 37 \\
\hline Self-inductance at $16 \mathrm{~T}(2$ apertures $)$ & $\mathrm{mH}$ & 570 \\
\hline Field margin on the load-line at $16 \mathrm{~T}$ & $\%$ & 14 \\
\hline Distance between aperture axes at $1.9 \mathrm{~K}$ & $\mathrm{~mm}$ & 250 \\
\hline Number of coil tums per aperture & - & 200 \\
\hline Cross-section of conductor $(2$ apertures $)$ & $\mathrm{cm}{ }^{2}$ & 133 \\
\hline Mass of the cold mass & $\mathrm{t}$ & 55 \\
\hline Field quality at injection $\left(b_{2}, b_{3}\right)$ at $2 / 3$ of \\
aperture radius & units & $<20$ \\
\hline Field quality at collision $\left(b_{2}, b_{3}\right)$ at $2 / 3$ of \\
aperture radius & units & $<3$ \\
\hline
\end{tabular}

magnet systems of both colliders are based on twin-aperture $16 \mathrm{~T}$ $\mathrm{Nb}_{3} \mathrm{Sn}$ dipole magnets with an aperture of $50 \mathrm{~mm}$. The main target parameters of the cos-theta baseline $\mathrm{Nb}_{3} \mathrm{Sn}$ dipole magnet design are provided in Table I.

The design, manufacturing and operation of such dipoles in accelerator quality in large quantities is a large step forward and requires a dedicated development program. The program prof-its largely from the experience gained through CERN's HighLuminosity LHC (HL-LHC) program with $\mathrm{Nb}_{3} \mathrm{Sn}$ magnets [1], [2]. Critical aspects identified as essential for succeeding in designing, cost-effectively manufacturing and reliably operating 16 $\mathrm{T}$ dipole magnets in large colliders are: (a) the improvement of the state of the art conductor performance towards $1500 \mathrm{~A} / \mathrm{mm}^{2}$ and a cost of $5 \mathrm{EUR} / \mathrm{kA} . \mathrm{m}$ at $16 \mathrm{~T}$ and $4.2 \mathrm{~K}$ com-pared to 1000 $\mathrm{A} / \mathrm{mm}^{2}$ and $20 \mathrm{EUR} / \mathrm{kA} . \mathrm{m}$ at $16 \mathrm{~T}$ and $4.2 \mathrm{~K}$ in the HL-LHC project (b) the design of cost-effective $16 \mathrm{~T}$ dipole magnets with adequate electromagnetic and structural designs, and (c) the improvement of training. These points are addressed in a worldwide collaboration through different programs.

In Europe the work started in 2013, after the approval of the CERN council, and is dedicated to achieving the target parameters of the FCC dipole magnets [3]. In the US the 2014 Particle Physics Project Prioritization Panel (P5) identified a critical need for high-field magnet R\&D focused on substantially increasing performance and reducing the cost per T.m, which triggered the US Magnet Development Program (MDP) [4]. The US program started in June 2016 with the goal of exploring the performance limits of $\mathrm{Nb}_{3} \mathrm{Sn}$ accelerator magnets, pursuing $\mathrm{Nb}_{3} \mathrm{Sn}$ conductor $\mathrm{R} \& \mathrm{D}$ towards increased performance and reduced cost and investigating fundamental aspects of magnet design and technology for substantially improving their performance and reducing magnet cost.
Both in the US and Europe the conductor development is seen as key. The CERN managed program is developed in three phases. In the first phase the focus is devoted to the in-crease of the critical current by $50 \%$ with respect to HL-LHC $\left(1500 \mathrm{~A} / \mathrm{mm}^{2}\right.$ at $4.2 \mathrm{~K}$ and $16 \mathrm{~T}$ ), maintaining a high residual resistivity ratio (RRR) of 150. Achieving this goal requires a major breakthrough and work on novel methods, as Artificial Pinning Centres (APC), grain refinement and architectures. In the second phase the conductor will be optimized for the reduction of magnetization, in particular at low fields, by acting on the effective filament diameter and possibly on APC. The third phase can be considered the preparation to industrialization, with focus on achieving long unit length $(5 \mathrm{~km})$ and competitive cost $(5 \mathrm{kEuro} / \mathrm{kAm}$ at $4.2 \mathrm{~K}$ and $16 \mathrm{~T}$ ). The US program is synergic to the CERN program and is tackling similar targets.

To be able to sustain the European magnet program until 2020 up to around $500 \mathrm{~km} \mathrm{Nb}_{3} \mathrm{Sn}$ wire of different diameters in the range of $0.7 \mathrm{~mm}$ to $1.1 \mathrm{~mm}$ will be procured from dif-ferent suppliers within the framework of CERN's technology companion conductor program. Moreover, CERN established bilateral agreements with collaborating institutes and compa-nies in Europe and Asia for conductor development and characterization namely with the High Energy Accelerator Research Organization KEK (Japan), the University of Geneva (Switzer-land), the University of Freiberg (Germany) and the companies Kiswire Advanced Technology (Korea), TVEL (Russia), Bruker (Germany), and Luvata (Finland).

In the US, conductor development for high field accelerator magnets is organized primarily through the Conductor Procurement and R\&D (CPRD) program, an element of the US Magnet Development Program (MDP) focusing on advancing LTS and HTS industrial conductors. For $\mathrm{Nb}_{3} \mathrm{Sn}$, the program focuses on determining the performance limits, future scalability, industrialization and cost reduction.

The design and technology development towards $16 \mathrm{~T}$ dipole magnets is carried out within the European Program EuroCirCol WP5 (2015-2019), CERN's FCC Magnet Technology Companion Program (started in 2015), and the U.S. Magnet Development Program (started in 2016, focused on general R\&D for high field accelerator magnet technology, consistent with the FCC goals).

Within EuroCirCol WP5, the program is focused on costeffective cos-theta, block-type and common-coil electromagnetic and structural designs and $\mathrm{Nb}_{3} \mathrm{Sn}$ strand and cable characterization. The work performed within this program is the base for the Conceptual Design Reports (CDRs) for FCC and HE-LHC.

Within CERN's FCC Magnet Technology Companion Program the following initiatives are pursued: the design and manufacture of flat-racetrack coils accessing the $16 \mathrm{~T}$ field range with margin, coil manufacturing and property characterization focused on enhancing the understanding of the irreversible degradation, the windability of Rutherford cables and the material characterization of $\mathrm{Nb}_{3} \mathrm{Sn}$ coil packs. In the last years these programs have been substantially complemented with bi-lateral collaboration agreements between CERN and institutes covering aspects not yet treated in other programs. CEA 
(Commissariat a' l'energie' atomique et aux energies' alternatives, France), INFN (Istituto Nazionale di Fisica Nucleare, Italy) and CIEMAT (Centro de Investigaciones Energeticas,' Medioambientales y Tecnologicas,' Spain) aim at designing and manufacturing $16 \mathrm{~T}$ block-type, cos-theta and common-coil dipole models, respectively. Other agreements have been established with Budker Institute of Nuclear Physics (Russia) on the design and manufacture of a $16 \mathrm{~T}$ dipole model; with the Paul Scherrer Institute (Switzerland) on the design of a CCT $16 \mathrm{~T}$ demonstrator and manufacture of an $11 \mathrm{~T}$ technology demonstrator; with EPFL (Ecole polytechnique fed'erale' de Lausanne, Switzerland) on $\mathrm{Nb}_{3} \mathrm{Sn}$ splices; with the ETHZ (Eidgenossische“ Technische Hochschule Zurich," Switzerland) on the characterization of impregnation systems for $\mathrm{Nb}_{3} \mathrm{Sn}$ coils; with the Tampere University of Technology (Finland) on quench protection of $\mathrm{Nb}_{3} \mathrm{Sn}$ magnets and the industrialization of the production of $16 \mathrm{~T} \mathrm{Nb}_{3} \mathrm{Sn}$ magnets and with the University of Patras (Greece) on compact mechanical structures for $16 \mathrm{~T}$ dipoles.

The U.S. Magnet Development Program is initially focused on a strong technology development effort, along with a twopronged approach towards high field magnet design and testing currently including: a) the design, fabrication and test of building a 4-layer $15 \mathrm{~T}$ cos-theta dipole demonstrator with an aperture of $60 \mathrm{~mm}$, which is scheduled to be tested in 2018; and b) the development and testing of the Canted Cosine-theta concept (CCT), initially through the design, manufacture and test of 2-layer $10 \mathrm{~T}$ magnets. The program will proceed towards higher-field concepts based on results from these first tests. On the technology front, areas of focus include a) detailed characterization and comparison of epoxies and interfaces b) development of characterization and novel diagnostics including quench antennae and passive and active acoustic instrumentation to advance the understanding and mitigation of training behavior, and c) advanced modeling techniques utilizing computer clusters and parallel computing to enable multiphysics modeling of magnet systems. Under the Small Business Innovation Research (SBIR) and Small Business Technology Transfer (STTR) programs of the US Department of Energy (DOE) [5] synergic developments on conductor and magnet technology are performed.

The CCT design is also being explored by PSI, where the R\&D focuses on particular design elements that render CCT magnets more compact and competitive in conductor usage, the ultimate goal being a 4-layer $16 \mathrm{~T}$ magnet.

The status of development is presented in the HE-LHC and FCC CDRs [6]. These CDRs are a major input for the Update of the European Strategy for Particle Physics (ESPP), which are planned to be approved by the CERN council in May 2020 after a bottom-up process that starts with the broad consultation of all stakeholders in Europe's particle physics community and culminates in a dedicated meeting of the European Strategy Group. In this review the ESPP for the next years is defined. It is planned to update the ESPP again in around five years.

The overall aim of these programs and collaborations is to enable the required technology development, design and manufacture of cost effective high-field $\mathrm{Nb}_{3}$ Sn dipole model mag-nets accessing the $16 \mathrm{~T}$ field range. The results and lessons learned will enable validating and testing the specific interesting characteristics of the different design options and allow a downselection of the design option, to be able to formulate a clear vision for the construction of long models (2023-2027) at the time of the next but one ESPP and for industry proto-types (20272032), pre-series magnets (2032-2036), and series magnets (2036-2041).

In this paper the main results of the work performed in the different programs is discussed. We believe that the work performed, will allow us to submit a credible $16 \mathrm{~T}$ dipole magnet CDR to the European Strategy Group.

\section{CONDUCTOR DEVELOPMENT AND PROCUREMENT}

Despite the short time span of the programs, high-performing $\mathrm{Nb}_{3} \mathrm{Sn}$ conductors have been already produced by new collaborating partner institutes and companies, achieving a $J_{c}$ performance in the order of the specification for HL-LHC [7]. Work performed on grain refinement and APC has shown very promising results, achieving the FCC specification in critical current density on small samples [8], [9]. To improve the training of magnets the addition of materials with high heat capacity $\left(\mathrm{Gd}_{2} \mathrm{O}_{3}\right)$ incorporated directly within the $\mathrm{Nb}_{3} \mathrm{Sn}$ wire is being investigated, preliminary results already indicate a much increased minimum quench energy (MQE) [10] and, thus, may allow significant reduction of magnet training.

As next step, the conductor optimization for the reduction of magnetization, in particular at low fields, by acting on the effective filament diameter and possibly on APC. After being able to produce wire with the required technical specification, an industrialization phase is planned focusing on achieving long unit length $(5 \mathrm{~km})$ and competitive cost $(5 \mathrm{EUR} / \mathrm{kA} . \mathrm{m}$ at $4.2 \mathrm{~K}$ and 16 T).

\section{Magnet Design}

Cos-theta [11], block type [12], common-coil [13], canted-cos theta (CCT) [14], [15] and cos-theta magnets with stress management [16] are being studied as design options for twinaperture dipole magnets accessing the $16 \mathrm{~T}$ field range. The electromagnetic design of the different design options is shown in Fig. 1. The cos-theta design was chosen as baseline design, as among these four optimized designs, it was found to be the most efficient in terms of amount of conductor used for a given integrated field strength and the same margin on the load-line; with respect to the conductor amount required for the cos-theta the block requires $3.7 \%$, the common-coil $25.4 \%$ and the CCT $27.7 \%$ more conductor. Each design option was optimized using the same assumptions in terms of conductor, load margin, field quality and quench. The relative difference in conductor usage may change if each design is allowed to optimize conductor for its own efficiency and quench performance.

The cos-theta was also the design option of choice for all colliders with SC magnets so far. Each of the alternative designs features specific interesting characteristics, which may have the potential to become competitive with the baseline cosine-theta design in terms of performance. Therefore, their designs are being fully developed and it is planned to explore them through model magnets. 


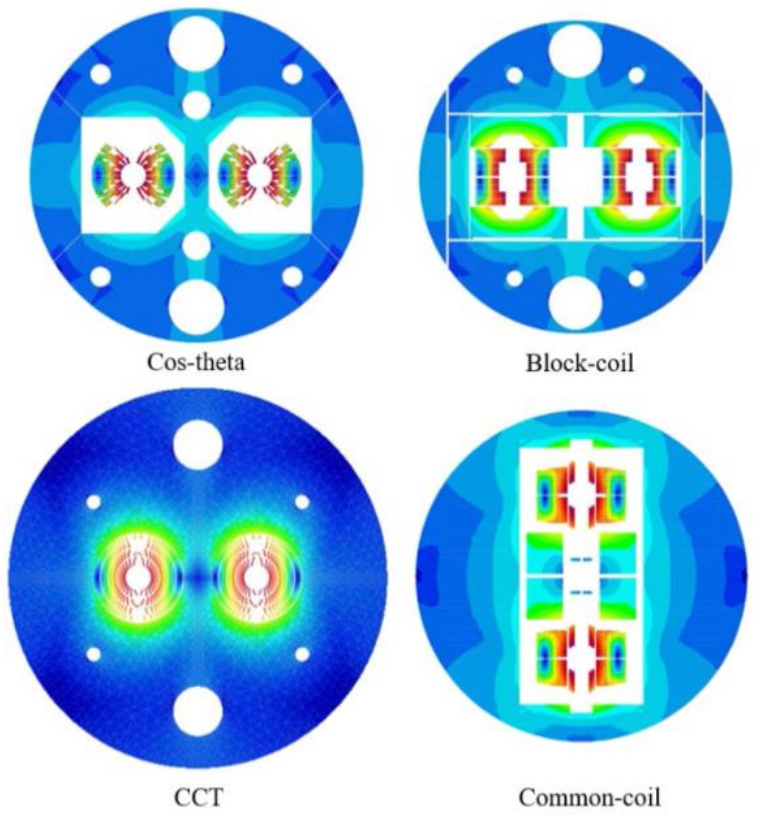

Fig. 1. Electromagnetic design of the different design options.

TABLE II

Baseline Target Parameters of the Conductor for the Main Dipoles

\begin{tabular}{ccc}
\hline Item & Unit & Value \\
\hline Critical current density at 16 T and 4.2 K & $\mathrm{A} / \mathrm{mm}^{2}$ & 1500 \\
\hline Strand diameter HF/LF conductor & $\mathrm{mm}$ & $1.1 / 0.7$ \\
\hline Filament diameter HF/LF conductor & $\mu \mathrm{m}$ & $20 / 20$ \\
\hline Cu/NoaCu HF/LF conductor & - & $0.8: 1 / 2.1: 1$ \\
\hline Number of strands HF/LF cable & - & $22 / 38$ \\
\hline Width of HF/LF cable & $\mathrm{mm}$ & $13.2 / 14$ \\
\hline $\begin{array}{c}\text { Keystone angel of HF/LF cable (only for } \\
\text { cos-theta design) }\end{array}$ & degree & 0.5 \\
\hline Average thickness of HF/LF cable & $\mathrm{mm}$ & $1.950 / 1.265$ \\
\hline
\end{tabular}

To keep the magnet size and mass within reasonable limits, it has been decided to accept a fringe field outside the cryostat of up to $0.1 \mathrm{~T}$, which is considered safe for other equipment. This decision allowed to reduce the cold mass diameter to $800 \mathrm{~mm}$ and to fit the cold mass into a cryostat with an outer diameter of 1250 $\mathrm{mm}$; compatible with an installation in both the FCC and HELHC. Studies are on-going to further optimize the structure towards compactness.

\section{A. Baseline Conductor Parameters}

Two distinct conductors are foreseen for the $16 \mathrm{~T}$ dipoles: a high-field (HF) conductor used for the inner coil and a low-field (LF) conductor used for the outer coil. The target parameters of the HF and LF conductor are summarized in Table II. It is assumed in the magnet design that the insulated cable can be submitted to pressures of up to $150 \mathrm{MPa}$ at ambient temperature and $200 \mathrm{MPa}$ when cold, without experiencing an irreversible degradation of its characteristics. Based on the information coming

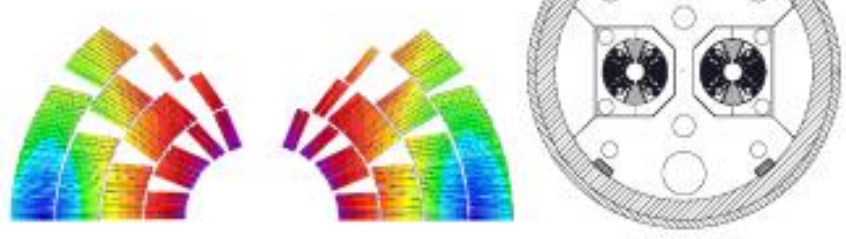

Fig. 2. FCC and HE-LHC baseline cross-section (left: Coil cross-section slightly left/right asymmetric to compensate $b_{2}$, right: cold mass with an outer diameter of $800 \mathrm{~mm}$ ).

from tailored experiments and from magnet tests, these values are considered to be challenging but realistic (see Section IV. A).

Due to the high $J_{\mathrm{c}}$, the large filament diameter and the large amplitude of a magnet cycle, the magnetization losses of these magnets have a considerable impact on the design of the cryogenic system (see next Section III. B). This limit can be respected with filament diameters up to around $20 \mu \mathrm{m}$, grain refinement, the introduction of APC, and with the optimization of the re-set current during the machine powering cycle. R\&D work for achieving small sub-elements with reasonable heat treatment cycles is planned.

\section{B. Baseline Dipole Design}

The baseline dipole is foreseen to be assembled in a helium tight cold mass (CM) structure, integrated in a cryostat: a cross section of the coil and cross-section is presented in Fig. 2.

The CM for the FCC main dipole (MD) is straight and has a total length between the two extremities of the beam pipe flanges of $15.8 \mathrm{~m}$ and a magnetic length of $14.069 \mathrm{~m}$. The CM for HELHC follows the beam's path and is therefore curved with a sagitta of around $9 \mathrm{~mm}$. The CM external diameter is of $800 \mathrm{~mm}$. Its cryostat structure is composed of a radiation shield, a thermal screen and a vacuum vessel. The CM is supported by three feet made from a composite material. All parts between the beam pipe and the shrinking cylinder (defining the outer envelope of the cold mass) are immersed in superfluid helium at atmospheric pressure and are cooled by a heat-exchanger tube. In the heat exchanger tube two-phase low-pressure helium circulates. The next temperature stage is that of the beam screen, cooled at a reference temperature of $50 \mathrm{~K}$, which also corresponds to the temperature level for cooling the thermal screen and the support posts. The fact that the additional intermediate temperature level used in the LHC, in the range of 4 to $20 \mathrm{~K}$ is missing, results in larger static losses from the cold mass and the support posts than in the LHC. The total heat loads of a cryodipole operating in steady state mode are estimated to be about $0.5 \mathrm{~W} / \mathrm{m}$ at $1.9 \mathrm{~K}$ and about $10 \mathrm{~W} / \mathrm{m}$ at $50 \mathrm{~K}$. The target losses during a full cycle from nominal field, down to injection and up to nominal field again, for which a large portion comes from the magnetization of the superconductor, are set to $5 \mathrm{~kJ} / \mathrm{m}$ at $1.9 \mathrm{~K}$ for the two apertures, such that the cryogenic system can reset the temperature within 2 $\mathrm{h}$. The design field of $16 \mathrm{~T}$ is generated by a current of $11,390 \mathrm{~A}$ in a coil which has a physical aperture of $50 \mathrm{~mm}$ and the distance between the axis of the two apertures is $250 \mathrm{~mm}$. 
Each MD aperture has 200 cable turns distributed in one upper and one lower coil, and each coil comprises two double layer (inner and outer) coils. Since the magnetic flux density varies considerably in the coil (it is much higher in the inner than in the outer coil), the design exploits the principle of grading (see below). The inner coil comprises 32 turns of a $0.5^{\circ}$ keystoned Rutherford cable, made from 22 strands of $1.1 \mathrm{~mm}$ diameter, the outer coil has 68 turns of a $0.5^{\circ}$ keystoned Rutherford cable, made from 38 strands of $0.7 \mathrm{~mm}$ diameter.

The current density in the outer coil is larger than that in the inner coil because the two coils are connected in series and the inner layers cable has a larger conductor area than the outer layers cable. This design exploits the concept of grading, which consists of increasing the current density where the magnet field is lower, resulting in a considerable saving of conductor for a given margin on the load-line, which for the FCC MD has been set to $14 \%$. The structure is based on the so-called key and bladders concept together with the use of an aluminum cylinder surrounded by a stainless steel welded shell. The aluminum shell provides an increase of coil loading, as required from assembly to the operational temperature and during magnet energization. This design choice, compared to a traditional collared magnet, allows keeping the stress at all steps (assembly, cool-down, powering to $16 \mathrm{~T}$ ) below the stipulated limits, at which the conductor would start degrading irreversibly. The stainless shell, in addition to increasing the stiffness of the structure, provides helium tightness, alignment and support for the magnet end covers.

Prior to installation each magnet will be cold tested. Depending on its training performance, the magnet may be also submitted to a thermal cycle to confirm that, once installed in the tunnel, the target to power the magnets to nominal field without experiencing training quenches can be achieved. Concerning magnetic measurements, a warm-cold correlation will be established based on the statistics on pre-series magnets, as it was successfully done for the LHC. All series magnets will be then magnetically measured warm and only a small percentage of them also measured cold.

\section{Quench Protection}

The magnet and its protection system are conceived to limit the hot spot temperature in the case of a quench to below $350 \mathrm{~K}$ and the peak voltage to ground in the coil below $2.5 \mathrm{kV}$. This voltage limit comprises up to $1.2 \mathrm{kV}$ due to the quench evolution in the magnet itself and up to $1.3 \mathrm{kV}$ from the circuit. The protection system can be based on the coupling-loss-induced quench method (CLIQ), on quench heaters alone or on a combination of both. On paper all options effectively protect the magnets within the above limits [17]. Experiments on the FCC models and prototypes will allow to understand in real conditions if CLIQ can be implemented with the required reliability and redundancy for every quench condition. For the reasons above, though it is believed that CLIQ has the potential to quench the entire magnet in $30 \mathrm{~ms}$ after the initiation of a quench (time delay), the $16 \mathrm{~T}$ magnets have been designed assuming a time margin of at least $40 \mathrm{~ms}$, which is compatible with the use of quench-heaters.

\section{Field Quality}

The field error naming convention follows the one adopted for the LHC [18]. The systematic field error values are deterministic and computed with ROXIE: they are made of a geometric contribution, a contribution coming from persistent currents and the effect of saturation of the ferromagnetic yoke (see Table I). The contribution of the persistent currents [19] has been computed considering the conductor characteristics of Table II.

The coils are slightly asymmetric to compensate for the $b_{2}$ component and to bring this component well below the target specification. Further optimization is on-going to passively correct the $b_{3}$ error from persistent current by using iron shims. The yoke shape will also be further optimized to minimize the saturation effects.

\section{E. Cost Estimate}

The magnet cost has been split in three main contributions: the conductor cost, the assembly cost, and the cost of the magnet parts [20]. The main contributor is the conductor cost, which is of about $670 \mathrm{kEUR} /$ magnet considering the FCC target conductor cost of $5 \mathrm{EUR} / \mathrm{kA} . \mathrm{m}$ at $4.2 \mathrm{~K}$ and $16 \mathrm{~T}$. This cost is between three and four times lower than the present cost considered for conductor procured for HL-LHC, noting that the expected $50 \% J_{\mathrm{c}}$ increase has a direct impact on the cost reduction. Due to the limited number of suppliers and limited demand on the market at this stage, this cost is considered the most uncertain.

The cost of the parts amount to $450 \mathrm{kEUR} / \mathrm{magnet}$, is based on present costs and is estimated to be solid as production can be performed by standard manufacturing industry.

The cost for the assembly has been set to $600 \mathrm{kEUR}$, which is about twice the cost of the assembly of the LHC magnets. This cost is dominated by the number of coils to be made in a magnet (twice than for the LHC) and by their increased complexity. The production will require a tailor-made production line and the final cost will depend on the degree of industrialization that can be achieved during the series production. A study on how to industrialize this production has therefore already been initiated with the University of Tampere (UoT), taking the present production for HL-LHC as test bed, first results are expected soon.

Mainly due to the uncertainty of the cost of the conductor, and also to the opportunities that a $\mathrm{R} \& \mathrm{D}$ program may provide to simplify the magnet manufacture, we believe that it is more reasonable to give a range than a given number, between 1.7 and 2.0 MEUR/magnet.

\section{MAGNET TECHNOLOGY DEVELOPMENT}

Magnet technology development is seen as key for succeeding in building $16 \mathrm{~T}$ dipole magnets. Dedicated tests and studies enable the possibility of exploring different critical aspects within shorter time and with lower cost compared to model magnets. 


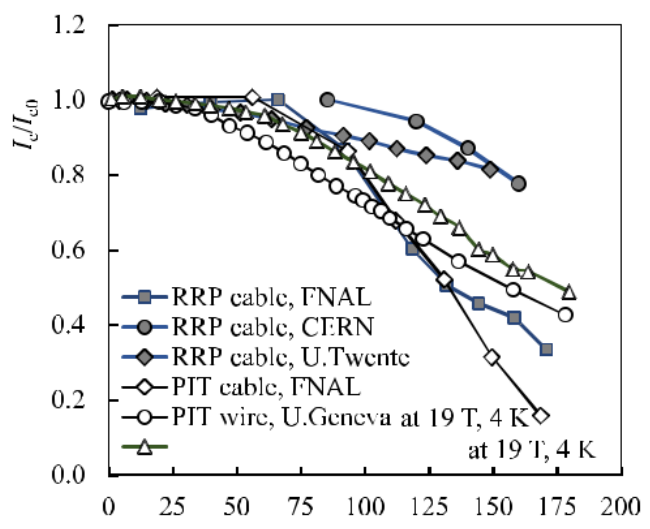

Fig. 3. Normalized $I_{\mathrm{C}}$ at $12 \mathrm{~T}$ and $4.2 \mathrm{~K}$ (unless otherwise stated in the legend) vs. transverse pressure on Rutherford cable face or on encased wire for epoxy impregnated samples.

The different initiatives and their preliminary results are discussed in this section.

\section{A. Effect of Transverse Pressure}

Transverse stress is the largest stress component in accelerator magnets: this shall be lower than the stress level producing critical current degradation on the brittle $\mathrm{Nb}_{3} \mathrm{Sn}$ conductor. Two components to critical current degradation can be distinguished: a reversible component, which is fully recovered when removing the load, and an irreversible and therefore permanent component. The irreversible limit is defined as the pressure leading to a $95 \%$ recovery of the initial $I_{\mathrm{c}}$, or $I_{\mathrm{c} 0}$, after unloading the sample. To determine $I_{\mathrm{C}}$ sensitivity to transverse pressure, electro-mechanical tests were performed on cables and encased wires.

The $I_{\mathrm{c}}$ degradation strongly depends on conductor technology, but also on sample preparation and setup design. The for-mer has an impact on possible stress concentration; the latter determines the sample's actual stress-strain state.

Fig. 3 shows the total $I_{\mathrm{C}}$ degradation at $4.2 \mathrm{~K}$ and $12 \mathrm{~T}$ (un-less otherwise indicated in legend), at transverse pressures up to $\sim 200$ $\mathrm{MPa}$, of epoxy-impregnated Rutherford cables and en-cased wires made of PIT and RRP [21]-[23]. Most of the load in this plot was applied cold. Older data (not shown) indicate that cables made of low- $J_{c}$ strands are less sensitive to transverse pressure than those made with state-of-the-art, high- $J_{c}$ strands. In some cases a stainless-steel core inside the cable also reduced pressure sensitivity.

Recent experiments performed at Fresca to measure the irreversible component with the load applied warm showed that the irreversible component is negligible, at least up to $150 \mathrm{MPa}$ [24].

\section{B. Coil Structural Characterization}

The knowledge of the structural properties of the coil is essential for the design process of the magnets in order to pre-dict the stress levels and to avoid excessive stress leading to degradation. The experimental determination of stress-strain curves measured in the three directions (transversal, axial, and radial) and made out of ten-stacks or samples cut from mag-net coils show an elastic-plastic behavior with hysteresis and ratcheting. In simulations typically a linear-elastic coil behavior is assumed. To determine the linear-elastic stiffness value different fitting methods are under discussion [25], [26], yielding for the same measurements results differing by up to around $50 \%$. Measurement procedures and fitting methods are under further investigation to produce representative results. It also remains an open question, how well the free-standing samples used in the studies reflect the mechanical behavior in a coil configuration.

As a part of the US MDP, the thermomechanical properties of cable stacks that represent the cable composites in the $15 \mathrm{~T}$ dipole demonstrator were studied. The tests included thermal contraction and strain-stress characterization under compressive load of a cable stack sample impregnated with CTD-101K. The measurements are consistent with the above reported results [27].

Neutron diffraction measurements at the Heinz Maier-Leibnitz Zentrum (MLZ) at the instrument STRESS-SPEC have been performed in order to better understand the effect of the epoxy resin on the $\mathrm{Nb}_{3} \mathrm{Sn}$ and copper lattice strain and stress evolution under compressive stress loading [28].

\section{Material Characterization}

A program for structural characterization of presently used materials for the HL-LHC magnet production [29] has been extended to explore also lower-cost wedge materials (CuSn8P,

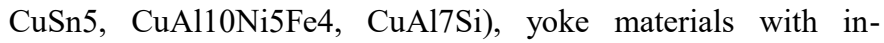
creased yield strength (microalloyed precipitation hardened ferritic steels) and ferromagnetic yoke material with reduced thermal contraction (FeNi36) which may allow the design and manufacture of more compact magnet yokes, if a larger fringe field is allowed. The measurement campaign includes the application of the heat treatment to the wedge material, and tensile, fracture toughness, and fatigue (yoke material only) testing at 4.2 $\mathrm{K}$. The thermal contraction from $300 \mathrm{~K}$ to $4.2 \mathrm{~K}$ was also measured for all materials. Preliminary results are reported in [30] and will be the base for structural design studies aiming at cost-effectiveness and compactness.

\section{Windability}

To be able to quantify under a well-defined, controlled and repeatable way the overall mechanical behavior of the Rutherford cable during the coil winding process a novel cable winding setup has been developed. In this set-up the Rutherford cable can be geometrically inspected during all winding steps. Three indicators for windability were defined for (1) the average envelope (distance between the reference winding surface and the cable), (2) strand pop-outs (a local change in the cable thickness caused by at least one strand coming out of the cable side plane) and (3) protrusion (a distance from the bottom support to the bottom of the cable). Establishing thresholds for the different indicators to allow good windability is still challenging and requires further work [31]. 


\section{E. Characterization of Impregnation Systems}

Recently built $\mathrm{Nb}_{3} \mathrm{Sn}$ magnets show typically a long training and difficulty in approaching ultimate currents. It is assumed that this long training is in part due to how the coil is manufactured (coil design and layout, winding, heat treatment, impregnation), and how the coil stress is managed (assembly process in the mechanical structure, design and operation modes of the structure). To establish a better understanding of the characteristics relevant to accelerator magnets of the different impregnation systems in terms of mechanical strength, adhesion, dielectric strength, ageing, and radiation hardness, flexural properties (ISO 178), the Charpy impact properties (ISO 179), and the compressive properties (ISO 604) at warm and cold are being measured within the framework of a CERN/ETHZ collaboration.

Significant work is also ongoing within the US MDP to evaluate and compare impregnation systems, with a focus on addressing magnet training. First results are expected to be published soon. Diagnostics are integrated into mechanical properties tests with the goal of identifying and correlating energy disturbances under various stress configurations (see Section IV.G).

\section{F. Internal Splicing}

Disregarding the winding layout the $\mathrm{Nb}_{3} \mathrm{Sn}$ accelerator magnets for FCC must be graded to minimize the strand cost. For the cos-theta design it is desirable and for the block design it is almost seen mandatory that the joints between grades are placed inside the winding pack to avoid lead extension and limit the mechanical discontinuity. To achieve splices with low resistance three methods are currently under study: ultrasonic welding (before HT), diffusion bonding (during HT), and soldering (after HT). Samples of the different methods are prepared and the splice resistance is measured in SULTAN at $10.9 \mathrm{~T}$ background field, $4.5 \mathrm{~K}$ operating temperature and a current up to $14 \mathrm{kA}$. First results are presented in [32].

\section{G. Instrumentation}

Within the US MDP, novel instrumentation is being developed to provide further insight into magnet behavior. A variety of quench antennae have been developed that aid in identifying quench initiation locations [33], and novel acoustic detection schemes are under development that enable detailed evaluation of mechanical disturbances in space and time, enabling 3dimensional quench initiation locations as well as a wealth of data on temporal distribution of mechanical energy depositions during magnet ramping [34], [35]. Many of these systems have been employed during recent testing of HL-LHC quadrupole prototype magnets.

\section{H. Coil R\&D}

At CERN a small-scale magnet R\&D program based on flat racetrack coils has been established to address questions related to the manufacture and design of high-field $\mathrm{Nb}_{3} \mathrm{Sn}$ magnets. The main parameters are summarized in Table III including the ultimate field Bultimate.
TABLE III

Main PaRAmeters of CERN R\&D FCC Magnets

\begin{tabular}{cccccc}
\hline \hline Parameter & Unit & SMC & RMC & ERMC & RMM \\
\hline OD & $\mathrm{mm}$ & 530 & 530 & 800 & 800 \\
\hline $\begin{array}{c}\text { Straight sec- } \\
\text { lion Iength }\end{array}$ & $\mathrm{mm}$ & 150 & 300 & 740 & 740 \\
\hline$B_{\text {ultimate }}$ & $\mathrm{T}$ & 14 & 16 & 18 & 18 \\
\hline Bore & - & $\begin{array}{c}\text { No } \\
\text { bore }\end{array}$ & No bore & No bore & $\begin{array}{c}50 \mathrm{~mm} \text { spac- } \\
\text { ing }\end{array}$ \\
\hline Mass strand & $\mathrm{kg}$ & 10 & 25 & 120 & 180 \\
\hline \hline
\end{tabular}

The CERN Short Model Coil (SMC) and Racetrack Model Coil (RMC) programs [36], [37], are considered an intermediate step between cable and magnet testing, providing a short turnover time and allowing the exploration of new ideas in a shorter time and at lower cost than proto-type or model magnets. In the context of FCC, the exist-ing SMC test bed is planned to be used for testing conductor variants, impregnation resins, for implementing and testing sliding and separating surfaces to investigate the impact on training and for developing high field internal splice technology.

As a logical sequel to the SMC/RMC program the CERN Enhanced Racetrack Model Coil (ERMC) and Racetrack Model Magnet (RMM) programs [38], [39] have been established as a test-bed for full size conductors reproducing full field and force conditions over a representative length, including transitions. ERMC is, alike to SMC and RMC, a flat racetrack coil without bore. RMM is made by using the same coils of ERMC, and inserting at the midplane location between two double pancake racetrack coils one (or two in a graded version) additional double pancake flat racetrack coil and is therefore considered representative for a full size test of a block-coil magnet, including a reproduction of the $2 \mathrm{D}$ cross section (with a cavity, no bore), and optimized ends $(0.5 \mathrm{~T}$ field drop). This program allows to validate the force and stress management (not considering the flared ends), transversal and longitudinal magnet loading and field quality in the straight part.

A first ERMC magnet is currently being produced to test the overall production process and different loading conditions. Future ERMC/RMM magnets are planned to explore conductor grading, conductor interfaces to pole and end-spacers, layer jumps and splices, and different heat treatment cycles and impregnation systems.

Fig. 4 shows a cross-section and a picture of the first ERMC coil. The first magnet is scheduled for test in summer 2019. The results and lessons-learned will be an important input, in particular for what concerns pre-loading conditions, and will give first information for the magnet prototypes on the choice of sliding planes, layer jump and splicing technology.

At PSI, a program to build an 11 T CCT technology demonstrator called CD1 (Canted Dipole 1) is on-going. CD1 features a reduced-volume winding former in order to increase the conductor efficiency. To compensate for the loss in mechanical rigidity a mechanical structure based on the bladder-and-key concept was adapted for the particular needs of CCT magnets. For manufacturing of CD1 and future magnets at PSI, $\mathrm{a} \mathrm{Nb}_{3} \mathrm{Sn}$ 

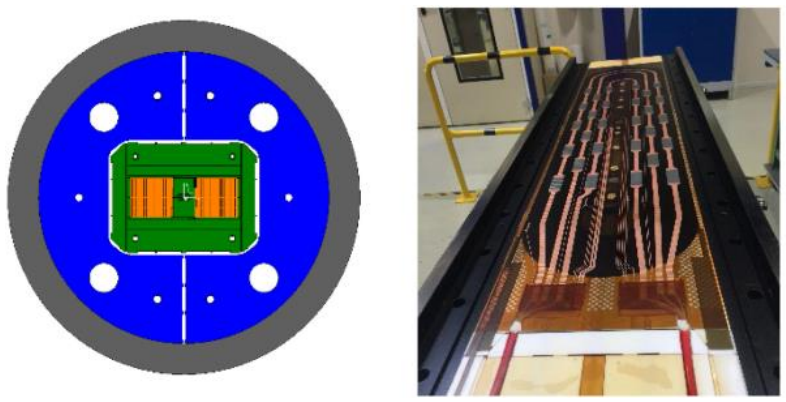

Fig. 4. Cross-section (left) and photo of a heat treated and impregnated ERMC coil.

coil manufacturing infrastructure has been established and successfully commissioned. The magnet components have been procured and are available. CD1 is foreseen to be tested during the first half of 2019. The magnet design was created in close coordination with the efforts at LBNL. In many details, the LBNL and PSI programs explore complementary solutions to open design challenges, while PSI adopts proven technologies from $L B N L$ in other areas.

The LBNL CCT effort [40], A core research element of the US MDP, is focused on developing the CCT technology in a systematic and scalable manner, so as to identify and address technical hurdles quickly. A series of two $\mathrm{Nb}$-Ti and two $\mathrm{Nb}_{3} \mathrm{Sn}$ magnets have been made to date; the last magnet, CCT4, reached $\sim 9.1 \mathrm{~T}$ in a $90 \mathrm{~mm}$ bore (around $86 \%$ of the short sample field), demonstrating basic feasibility of the concept, but exhibiting significant training ( $\sim 100$ training quenches). A next version of the magnet (CCT5) has been fabricated, incorporating a number of modifications designed to impact training performance, leveraging analysis and diagnostics data [41]. The key modification from previous magnets is the development of a new assembly method which uses epoxy filled shims between the magnet layers and the epoxy, Mix61, replacing the usual CTD-101K. The assembly is performed while applying external force to deform the outer Aluminium shell. This process is used to apply directional bending to the magnet and allows for better control of the contact interfaces.

\section{MAGNET MODELS}

Models of different design options (CEA (block-type, around 10 coils [42]), INFN (cos-theta, 6 coils), CIEMAT (common-coil, 6 coils), PSI (CCT) and BINP (different designs under study)) are being considered to be built and tested in the period until 20222025.

In the US one $15 \mathrm{~T}$ dipole demonstrator (Fig. 5, left) with an aperture of $60 \mathrm{~mm}$ is currently being built. A full set of 4 coils (two inner and two outer coils) are finished. The mechanical structure (Fig. 5, right) was assembled, tested with "dummy" coils and the initial values of the magnet prestress is deter-mined. Several LN2 tests of the mechanical structure com-ponents were performed and additional optimization is carried out. The final assembly is in progress and the cold test is foreseen for this year [43].
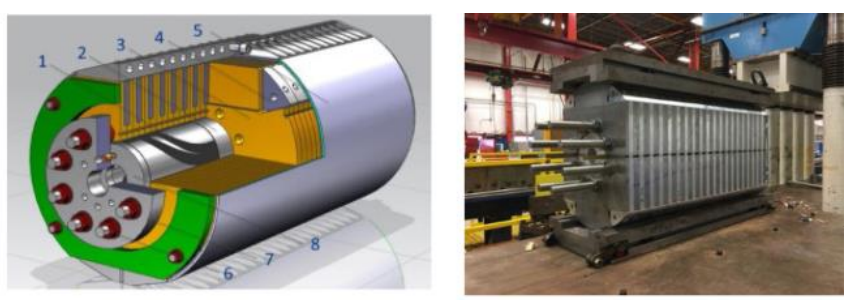

Fig. 5. The U.S. MDP $15 \mathrm{~T}$ dipole demonstrator (left) and mechanical structure (right).

\section{CONCLUSION}

An integrated program to develop $16 \mathrm{~T} \mathrm{Nb}_{3} \mathrm{Sn}$ dipole mag-nets for particle accelerators is being pursued under the push of the FCC study through a European Program (EuroCirCol), a technological program coordinated by CERN and a US Magnet Development Program since the years 2014-2015. Such large colliders, equipped with $16 \mathrm{~T} \mathrm{Nb}_{3} \mathrm{Sn}$ dipole magnets, call for the improvement of the state of the art conductor performance and the reduction of its cost, the design of innovative cost-effective dipole magnets with adequate electromagnetic and structural designs, and the improvement of training and approaching operating fields of $85-90 \%$ of their short sample limit. In the mentioned programs, building up on past experience, these points are addressed with priority. The results obtained so far allow us to compile CDRs with a credible technical proposal for the FCC and HE-LHC studies.

As next step, the design and manufacture of $16 \mathrm{~T}$ model magnets of different types and configurations is already under way or being organized. We believe that the work performed in these initiatives will build-up the experience for drawing a technical design report in the coming years for both FCC and HELHC.

\section{REFERENCES}

[1] D. Schoerling and A. V. Zlobin, Eds. $\mathrm{Nb}_{3} \mathrm{Sn}$ Accelerator Magnets - Designs, Technologies and Performance. Springer Nature, Jun. 2019, to be published, doi: 10.1007/978-3-030-16118-7.

[2] O. Bruning" and L. Rossi, The High Luminosity Large Hadron Collider: The New Machine for Illuminating the Mysteries of Universe. Singapore: World Scientific, 2015.

[3] D. Schoerling et al., "Strategy for superconducting magnet development for a future hadron-hadron circular collider at CERN," Proc. PoS (EPSHEP) 517, Vienna, Austria, 2015, doi: 10.22323/1.234.0517.

[4] S. Gourlay, A. V. Zlobin, S. Prestemon, D. C. Larbalestier, and L. Cooley, "The U.S. magnet development program plan," Lawrence Berkeley Nat. Lab., Berkeley, CA, USA, Jun. 2016.

[5] U.S. Department of Energy, Office of Science, Small Business Innovation Research (SBIR) and Small Business Technology Transfer (STTR), Research Areas and Impact, Mar. 2019. [Online]. Available: https:// science.energy.gov/sbir/research-areas-and-impact/

[6] Future Circular Collider, Conceptual Design Report, Jan. 2019. [Online]. Available: https://fcc-cdr.web.cern.ch

[7] A. Ballarino et al., "The CERN FCC conductor development pro-gram: A worldwide effort for the future generation of high-field magnets," IEEE Trans. Appl. Supercond,, to be published, doi: 10.1109/TASC.2019.2896469.

[8] X. Xu, X. Peng, J. Rochester, M. Sumption, and M. Tomsic, “Achievement of FCC specification in critical current density for Nb3Sn superconductors with artificial pinning centers," [Online]. Available: https://arxiv.org/abs/1903.08121

[9] F. Buta et al., "Properties and microstructure of binary and ternary $\mathrm{Nb}_{3} \mathrm{Sn}$ superconductors with internally oxidized $\mathrm{ZrO} 2$ nanoparticles," presented at the Appl. Supercond. Conf., Seattle, WA, USA, Oct. 28 Nov. 2, 2018, Paper 1MPo2A-06. 
[10] X. Xu et al., "Improvement of stability of $\mathrm{Nb}_{3} \mathrm{Sn}$ conductors and magnets by increasing specific heat," Supercond. Sci. Technol., vol. 31, no. 3. [Online]. Available: https://iopscience.iop.org/article/10.1088/ 13616668/aaa5de/meta

[11] R. Valente et al. "Electromagnetic design of a $16 \mathrm{~T} \cos \theta$ bending dipole for the future circular collider," IEEE Trans. Appl. Supercond., to be published, doi: 10.1109/TASC.2019.2901604.

[12] M. Segreti et al., "2-D and 3-D design of the bock-coil dipole option for the future circular collider," IEEE Trans. Appl. Supercond., vol. 29, no. 5, Aug. 2019, Art. no. 4000404.

[13] F. Toral, J. Munilla, and T. Salmi, "Magnetic and mechanical design of a 16 T common coil dipole for an FCC," IEEE Trans. Appl. Supercond., vol. 28 , no. 3, Apr. 2018, Art. no. 4004305.

[14] G. Montenero et al., "Coil manufacturing process of the first 1-m-long canted-cosine-theta (CCT) model magnet at PSI," IEEE Trans. Appl. Su-percond., vol. 29, no. 5, Aug. 2019, Art. no. 4002906.

[15] D. Arbelaez et al., "Design and test results of the $\mathrm{Nb}_{3} \mathrm{Sn}$ canted-cosineTheta dipole magnet CCT5," presented at the Appl. Supercond. Conf., Seattle, WA, USA, Oct. 28-Nov. 2, 2018, Paper 2LOr1B-04.

[16] A. V. Zlobin, J. R. Carmichael, V. V. Kashikhin, and I. Novitski, "Conceptual design of a $17 \mathrm{~T} \mathrm{Nb}_{3} \mathrm{Sn}$ accelerator dipole magnet," in Proc. 9th $I P A C$, Vancouver, BC, Canada, 2018. pp. 2742-2744.

[17] T. Salmi et al., "Quench protection of the $16 \mathrm{~T} \mathrm{Nb}_{3} \mathrm{Sn}$ dipole magnets de-signed for the Future Circular Collider," presented at the Appl. Supercond. Conf., Seattle, WA, USA, Oct. 28-Nov. 2, 2018, Paper 3LPo2E-03.

[18] R. Wolf, "Field error naming conventions for the LHC, LHC-M-ES0001, EDMS 90250," 2001, Engineering Specification.

[19] S. I. Bermudez, "Persistent currents magnetization effects in the $16 \mathrm{~T}$ main dipoles for the future circular collider," Technical note, EDMS: 2036614, 2018.

[20] D. Schoerling et al., "Considerations on a cost model for high-field dipole arc magnets for FCC," IEEE Trans. Appl. Supercond., vol. 27, no. 4, Jun. 2017, Art. no. 4003105.

[21] J. T. Troitino et al. " "Critical current degradation of $\mathrm{RRP} \mathrm{Nb}_{3} \mathrm{Sn}$ strands under applied transversal loads," presented at the Appl. Supercond. Conf., Seattle, WA, USA, Oct. 28-Nov. 2, 2018, Paper 1MPo2A-09.

[22] P. Gao et al., "Transverse pressure dependence of critical current in RRP and PIT type $\mathrm{Nb}_{3} \mathrm{Sn}$ Rutherford cables for use in future accelerator mag-nets," presented at the Appl. Supercond. Conf., Seattle, WA, USA, Oct. 28-Nov. 2, 2018, Paper 3LPo1D-09.

[23] C. Senatore et al., "Scaling behavior of the critical current under transverse stress in RRP and PIT $\mathrm{Nb}_{3} \mathrm{Sn}$ wires," presented at the Appl. Supercond. Conf., Seattle, WA, USA, Oct. 28-Nov. 2, 2018, Paper 2MOr1 A-02.

[24] P. Ebermann et al., "Irreversible degradation of $\mathrm{Nb}_{3} \mathrm{Sn}$ Rutherford cables due to transverse compressive stress at room temperature," Supercond. Sci. Technol., vol. 31, May 2018, Art. no. 065009.

[25] F. Wolf et al., "Effect of epoxy volume fraction on the stiffness of $\mathrm{Nb}_{3} \mathrm{Sn}$ Ruther-ford cable stacks," IEEE Trans. Appl. Supercond., to be published, doi: 10.1109/TASC.2019.2899497.

[26] J. L. R. Fernandez et al., "Characterization of the mechanical properties of impregnated $\mathrm{Nb}_{3} \mathrm{Sn}$ coils," IEEE Trans. Appl. Supercond., to be published, doi: 10.1109/TASC.2019.2897959.

[27] P. Li, S. Krave, and A. Zlobin, "Study of thermomechanical properties of the epoxy-impregnated cable composite for a $15 \mathrm{~T} \mathrm{Nb}_{3} \mathrm{Sn}$ dipole demonstrator," IOP Conf. Se.: Materials Sci. Eng., vol. 279, 2017, Art. no. 012020
[28] F. Wolf et al., "Effect of applied compressive stress and impregnation material on internal strain and stress state in $\mathrm{Nb}_{3} \mathrm{Sn}$ Rutherford cable stacks," IEEE Trans. Appl. Supercond., vol. 29, no. 5, Aug. 2019, Art no. 8400605, doi: 10.1109/TASC.2019.2893495.

[29] C. Scheuerlein, F. Lackner, F. Savary, B. Rehmer, M. Finn, and P. Uhlemann, "Mechanical properties of the HL-LHC $11 \mathrm{~T} \mathrm{Nb}_{3} \mathrm{Sn}$ magne constituent materials," IEEE Trans. Appl. Supercond., vol. 27, no. 4, Jun. 2017, Art. no. 4003007

[30] I. S. Fernandez and I. Sanchez, EDMS 2019015, Oct. 2018.

[31] D. Pulikowski, F. Lackner, C. Scheuerlein, F. Savary, D. Tommasini, and M. Pajor, "Windability tests of $\mathrm{Nb}_{3} \mathrm{Sn}$ Rutherford cables for HL-LHC and FCC," IEEE Trans. Appl. Supercond., vol. 28, no. 3, Apr. 2018, Art. no. 4003905

[32] M. Kumar et al., " $\mathrm{Nb}_{3} \mathrm{Sn}$ Rutherford cable splices for graded high field accelerator magnets," presented at the Appl. Supercond. Conf., Seattle, WA, USA, Oct. 28-Nov. 2, 2018, Paper 1LOr1D-04.

[33] M. Marchevsky et al., "Axial-field magnetic quench antenna for the super-conducting accelerator magnets," IEEE Trans. Appl. Supercond., vol. 25, no. 3, Jun. 2015, Art. no. 9500605.

[34] M. Marchevsky, X. Wang, G. Sabbi, and S. O. Prestemon, "Acoustic de-tection in superconducting magnets for performance characterization and diagnostics," presented at the Workshop on Accelerator Magnet, Super-conductor, Design and Optimization, Geneva, Switzerland, Jan. 15-16, 2013. arxiv.org. 16-Jan-2014.

[35] M. Marchevsky and S. A. Gourlay, "Acoustic thermometry for detecting quenches in superconducting coils and conductor stacks," Appl. Phys. Lett., vol. 110, no. 1, 2017, Art. no. 012601.

[36] E. Fornasiere et al., "Status of the activities on the $\mathrm{Nb}_{3} \mathrm{Sn}$ dipole SMC and of the design of the RMC," IEEE Trans. Appl. Supercond., vol. 23 , no. 3, Jun. 2013, Art. no. 4002308, doi: 10.1109/TASC.2013.2244932.

[37] J.-C. Perez et al., "16 $\mathrm{T} \mathrm{Nb}_{3} \mathrm{Sn}$ racetrack model coil test re-sult," IEEE Trans. Appl. Supercond., vol. 26, no. 4, Jun. 2016, Art. no. 4004906, doi: 10.1109/TASC.2016.2530684.

[38] S. Izquierdo Bermudez, R. Ortwein, J. C. Perez, and E. Rochepault, "De-sign of ERMC and RMM, the base of the $\mathrm{Nb}_{3} \mathrm{Sn} 16 \mathrm{~T}$ magnet development at CERN," IEEE Trans. Appl. Supercond., vol. 27, no. 4 Jun. 2017, Art. no. 4002004

[39] E. Rochepault, S. Izquierdo Bermudez, J. C. Perez, D. Schoerling, and D. Tommasini, "3-D magnetic and mechanical design of coil ends for the racetrack model magnet RMM," IEEE Trans. Appl. Supercond., vol. 28, no. 3, Apr. 2018, Art. no. 4006105.

[40] S. Caspi et al., "Design of a canted-cosine-theta superconducting dipole magnet for future colliders," IEEE Trans. Appl. Supercond., vol. 27, no. 4, Jun. 2017, Art. no. 4001505.

[41] D. Arbelaez et al., "Design and test results of the $\mathrm{Nb}_{3} \mathrm{Sn}$ canted-cosinetheta dipole magnet CCT5," presented at the Appl. Supercond. Conf. Seattle, WA, USA, Oct. 28-Nov. 2, 2018, Paper 2LOr1B-04.

[42] H. Felice et al., "F2D2: A block-coil short-model dipole toward FCC," IEEE Trans. Appl. Supercond., vol. 29, no. 5, Aug. 2019, Art. no. 4001807, doi: 10.1109/TASC. 2019.2897054.

[43] A. Zlobin et al., "Development of a $15 \mathrm{~T} \mathrm{Nb}_{3} \mathrm{Sn}$ dipole demonstrator by MDP," presented at the Appl. Supercond. Conf., Seattle, WA, USA, Oct. 28-Nov. 2, 2018, Paper 1LOr1D-02. 